\title{
A SINGULAR INITIAL VALUE PROBLEM TO CONSTRUCT DENSITY-EQUALIZING MAPS
}

\author{
A. AVINYÓ ${ }^{1}$, J. SOLÀ-MORALES ${ }^{2}$, AND M. VALÈNCIA $^{2}$
}

\begin{abstract}
The diffusion-based algorithm to produce density-equalizing maps interprets diffusion as an advection process. This algorithm uses the dynamics of a flow that is defined by an initial value problem that turns out to be very singular at the initial time. The singularities appear when the initial density has line or angle discontinuities, which is always the case, for example, in area cartogram maps. This singular initial value problem is analyzed mathematically in this paper and the conclusion is that despite these singularities, it has a unique solution. This justifies the extensive numerical use of this algorithm in the recent years. The techniques presented in this paper use both partial and ordinary differential equations estimates.
\end{abstract}

\section{INTRODUCTION}

For a given bounded domain $\Omega \subset \mathbb{R}^{n}$ and a density function $\rho: \bar{\Omega} \rightarrow \mathbb{R}^{+}$such that $\inf _{\bar{\Omega}} \rho>0$ and $\int_{\Omega} \rho=|\Omega|$, we proposed (see [2] and [3]) an algorithm to produce a diffeomorphism $\boldsymbol{\Phi}: \bar{\Omega} \rightarrow \bar{\Omega}$ that satisfies

$$
\operatorname{det}(D \boldsymbol{\Phi})(x)=\rho(x) .
$$

This prescribed Jacobian equation had been used in several problems of mathematical physics, such as the problem of equilibrium of gases considered in Section A.2.3 of the book of Dacorogna [7] or generating random distributions of particles on a domain with a given probability density (Russo [15]).

Independently of [2] and [3], the same algorithm was proposed by Gastner and Newman [9] to focus on the construction of geographical cartograms, maps of countries or regions where each region would be deformed to have an area proportional to a given quantity of economic or social interest, with the aim of building a reliable and efficient numerical method to produce them (see also [8] for extensive use of this method). More recently this algorithm has been used to construct non-uniform coverage of a planar region by sensor networks (Lekien and Leonard [13]). From a theoretical point of view, Carrillo and Lisini [6] have used a nonlinear diffusion version of this algorithm to study the asymptotic behavior of the solutions of some systems of nonlinear partial differential equations.

The algorithm is based on the idea that diffusion is a process that evenly distributes an arbitrary initial density $\rho$, and that the diffusion equation

$$
\frac{\partial}{\partial t} u(t, \mathbf{x})=\Delta u(t, \mathbf{x})
$$

Date: May 13, 2011.

1 Departament d'Informàtica i Matemàtica Aplicada, Universitat de Girona, Campus de Montilivi, 17071 Girona, Spain.

2 Departament de Matemàtica Aplicada 1, Universitat Politècnica de Catalunya, Av. Diagonal 647, 08028 Barcelona, Spain.

Partially supported by grants from the Spanish Government (MTM2008-06349-C03-01) and from the Catalan Government (2009SGR345) . 
can be written as an advection equation

$$
\frac{\partial}{\partial t} u(t, \mathbf{x})+\nabla \cdot(u(t, \mathbf{x}) \mathbf{V}(t, \mathbf{x}))=0
$$

if one uses as a velocity field $\mathbf{V}=-\nabla u / u$. Then, the map $\Phi$ of (1.1) is obtained by solving first (1.2) with homogeneous Neumann boundary conditions and initial condition $u(0, \mathbf{x})=\rho(\mathbf{x})$. After this, one has to solve the initial value problem for the ordinary differential equation

$$
\mathbf{x}^{\prime}(t)=\frac{-\nabla u(t, \mathbf{x}(t))}{u(t, \mathbf{x}(t))}
$$

with $\mathbf{x}(0)=\mathbf{x}_{0} \in \Omega$. And, finally, one defines $\Phi\left(\mathbf{x}_{0}\right)=\lim _{t \rightarrow \infty} \mathbf{x}(t)$ (see [3]).

The main problem for this algorithm to be well defined turns out to be the uniqueness of the solution of (1.4) near $t=0$, and, as a consequence, continuity with respect to the initial state. As

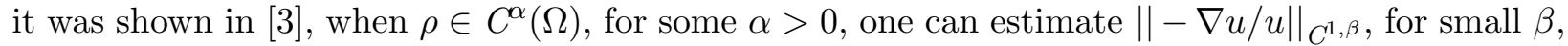
rather than $\operatorname{Lip}(-\nabla u / u)$. More exactly, one can use the maximum principle and the inequality

$$
\|\nabla u(t, \cdot)\|_{C^{1, \beta}} \leq \frac{C}{t^{1-(\alpha-\beta) / 2}}\|\rho(\cdot)\|_{C^{\alpha}}
$$

for the solution of (1.2) (see Belonosov [5] and Mora [12]). Then, when $0<\beta<\alpha, \operatorname{Lip}(-\nabla u / u$ ) is $L^{1}([0, T])$ and the situation is quite analogous to the weakly singular Volterra operators that appear in the semigroup approach to semilinear parabolic equations (see Henry [11]).

But, in the practical case of geographical cartograms, the class of admissible densities $\rho$ should include piecewise constant functions with discontinuities along curves and corners. In this discontinuous case, since $u(t, \mathbf{x}) \rightarrow \rho(\mathbf{x})$ as $t \rightarrow 0$, the ratio $\nabla u(t, \mathbf{x}) / u(t, \mathbf{x})$ will have obvious singularities when $t \rightarrow 0$. The study of these singularities is the goal of this paper.

From the viewpoint of asymptotic analysis, the previous considerations would lead to define the new stretched variables $\mathbf{x}^{\prime}=\left(\mathbf{x}-\mathbf{x}_{0}\right) / \varepsilon$ and $t^{\prime}=t / \varepsilon^{2}$, and to let $\varepsilon \rightarrow 0$ when $\mathbf{x}_{0}$ is a discontinuity point of $\rho$. The two equations (1.2) and (1.4) remain invariant by this change of variables. This is a well-known fact for the diffusion equation, but it is also very easy to see for the ordinary differential equation (1.4). The effect of letting $\varepsilon \rightarrow 0$ would only mean that the initial density $\rho$ is now defined in the whole plane and it is a function that is constant on sectors.

So, the aim of this paper is to study the singular initial value problem $(1.4)$ when $u(t, x)$ is the solution of (1.2), the domain is all the plane and the initial data $\rho$ is a positive piecewise constant on sectors function. We concentrate on the most difficult part, namely uniqueness. Some particular and simpler cases were studied in our previous publication [4]. The previous calculations are the origin of this work in the sense that gave us confidence on the fact that the effect of these singularities would not be to break the uniqueness of solutions.

\section{MAIN RESUltS}

In this section we present three theorems. The first one is the main result of the paper. The other two, that are needed to prove the first, are also interesting by themselves.

Definition 1. We will say that a function $\rho \in L^{\infty}\left(\mathbb{R}^{2}\right)$ is positive piecewise constant on sectors if there are strictly positive constants $C_{1}, C_{2}, \ldots, C_{k}$ and angles $\theta_{1}<\theta_{2}<\cdots<\theta_{k+1}=\theta_{1}+2 \pi$ such that $\rho(\mathbf{x})=C_{i}$ for all $\mathbf{x}$ with $\theta_{i}<\arg (\mathbf{x})<\theta_{i+1}$ and for all $i=1,2, \ldots, k$. 
Theorem 1. Let $\rho$ be a positive piecewise constant on sectors function and $u(t, \mathbf{x})$ be the solution to the linear heat equation in $\mathbb{R}^{2}$ with initial condition $\rho$. Then, for all $\mathbf{x}_{0} \in \mathbb{R}^{2}$, the initial value problem for the ordinary differential equation

$$
\left\{\begin{aligned}
\mathbf{x}^{\prime}(t) & =\frac{-\nabla u(t, \mathbf{x}(t))}{u(t, \mathbf{x}(t))}, \quad t>0 \\
\lim _{t \rightarrow 0^{+}} \mathbf{x}(t) & =\mathbf{x}_{0}
\end{aligned}\right.
$$

has at most one solution.

By using that the function $\rho$ is a positive piecewise constant on sectors and the symmetry properties of the heat equation, we will see that the differential equation in (2.1) has the particular form

$$
\mathbf{y}^{\prime}(t)=\frac{1}{\sqrt{t}} \mathbf{F}\left(\frac{\mathbf{y}(t)}{\sqrt{t}}\right),
$$

where $\mathbf{F}$ is a bounded vector function independent of time. So, it is quite clear that the Lipschitz constant of the right hand can be like $1 / t$. For this reason, in the proof of Theorem 1, we will need the following non-standard uniqueness theorem which is also interesting by itself. This theorem is inspired in the one-sided generalization of Nagumo's Criterion [14] due to Hartman (see Exercise 6.8. of [10]). For an extensive list of other uniqueness results for ordinary differential equations and systems see also Agarwal and Lakshmikantham [1]

Theorem 2. Let $\mathbf{G}(t, \mathbf{y})$ be a continuous function in $(0, T) \times \mathbb{R}^{n}$ and let there be three constants $M>0$ and $0<\beta<\alpha<1$ such that:

$$
\begin{gathered}
|\mathbf{G}(t, \mathbf{y})| \leq \frac{M}{t^{1-\alpha}}, \\
\left(\mathbf{G}\left(t, \mathbf{y}_{1}\right)-\mathbf{G}\left(t, \mathbf{y}_{2}\right)\right) \cdot\left(\mathbf{y}_{1}-\mathbf{y}_{2}\right) \leq \beta \frac{\left|\mathbf{y}_{1}-\mathbf{y}_{2}\right|^{2}}{t},
\end{gathered}
$$

for all $t>0$ and $\mathbf{y}_{1}, \mathbf{y}_{2} \in \mathbb{R}^{n}$. Then, the initial value problem

$$
\left\{\begin{aligned}
\mathbf{y}^{\prime}(t) & =\mathbf{G}(t, \mathbf{y}), \quad t>0, \\
\lim _{t \rightarrow 0^{+}} \mathbf{y}(t) & =\mathbf{y}_{0},
\end{aligned}\right.
$$

has at most one solution.

We will use this theorem only for $\alpha=1 / 2$. For this case, the inequality (2.4) of Theorem 2 for $G(t, \mathbf{x})=-\nabla u(t, \mathbf{x}(t)) / u(t, \mathbf{x}(t))$, where $u(t, \mathbf{x})$ is the solution of the heat equation in the plane, follows from the next theorem

Theorem 3. Let $\rho \in L^{\infty}\left(\mathbb{R}^{n}\right)$ such that $\inf _{\mathbb{R}^{n}} \rho>0$, and let $u(t, \mathbf{x})$ be the solution to the linear heat equation in $\mathbb{R}^{n}$ with initial condition $\rho$. Then, the following two properties hold:

a) If $\boldsymbol{\xi} \in \mathbb{R}^{n}$ is a unit vector, then

$$
\left(D_{\boldsymbol{\xi}}\right)^{2}(-\ln u(t, \mathbf{x}))<\frac{1}{2 t}
$$

for all $t>0$ and all $\mathbf{x} \in \mathbb{R}^{n}$.

b) If $n=2$ and $\rho$ is a positive piecewise constant on sectors function, then there is a number $\beta$ with $0<\beta<1 / 2$ such that the inequality (2.6) for $t=1$ can be improved to

$$
\left(D_{\boldsymbol{\xi}}\right)^{2}(-\ln u(1, \mathbf{x})) \leq \beta .
$$




\section{Proof of Theorem 2}

For a solution $\mathbf{y}(t)$ of $(2.5)$ we have the integral representation

$$
\mathbf{y}(t)=\mathbf{y}_{0}+\int_{0}^{t} \mathbf{G}(s, \mathbf{y}(s)) d s
$$

and, because of (2.3), it will satisfy

$$
\left|\mathbf{y}(t)-\mathbf{y}_{0}\right| \leq \frac{M}{\alpha} t^{\alpha}
$$

for all $t \in(0, T)$. Suppose now we have two different solutions $\mathbf{y}_{1}(t)$ and $\mathbf{y}_{2}(t)$. Then, by using (2.4), we would have, for $t>0$,

$$
\begin{aligned}
\frac{\left(\mathbf{y}_{2}(t)-\mathbf{y}_{1}(t)\right)^{2}}{t^{2 \alpha}} & =\frac{2}{t^{2 \alpha}} \int_{0}^{t}\left(\mathbf{G}\left(s, \mathbf{y}_{2}(s)\right)-\mathbf{G}\left(s, \mathbf{y}_{1}(s)\right)\right) \cdot\left(\mathbf{y}_{2}(s)-\mathbf{y}_{1}(s)\right) d s \\
& \leq \frac{2 \beta}{t^{2 \alpha}} \int_{0}^{t} \frac{\left(\mathbf{y}_{2}(s)-\mathbf{y}_{1}(s)\right)^{2}}{s} d s .
\end{aligned}
$$

Because of (3.2), the quantity

$$
Q=\sup _{0<t \leq T} \frac{\left(\mathbf{y}_{2}(t)-\mathbf{y}_{1}(t)\right)^{2}}{t^{2 \alpha}}
$$

is finite and, from the previous inequality, we get

$$
Q \leq \sup _{0<t \leq T} \frac{2 \beta}{t^{2 \alpha}} \int_{0}^{t} s^{2 \alpha-1} Q d s=\frac{\beta}{\alpha} Q
$$

which implies $Q=0$ since $\beta<\alpha$.

Remark 1. We now present a counterexample of Theorem 2 with $\alpha=\beta=1 / 2$. Let $G \in \mathcal{C}^{1}(\mathbb{R}, \mathbb{R})$ be a bounded function such that $G(x)=x / 2$ when $-1 \leq x \leq 1$ and $G^{\prime}(x) \leq 1 / 2$ for all $x$. Let us consider the ordinary differential equation

$$
x^{\prime}=\frac{1}{\sqrt{t}} G\left(\frac{x}{\sqrt{t}}\right) .
$$

This equation satisfies the hypotheses of the previous Theorem except $\beta<\alpha$, but one can observe that the following is a family of forward solutions

$$
x(t)=C \sqrt{t}, \quad-1<C<1, \quad 0 \leq t<\infty
$$

and all of them have the same initial value $x(0)=0$.

\section{Proof of Theorem 3}

Without loss of generality, let us take $\boldsymbol{\xi}$ as the unit vector in the direction of the $x_{1}$ coordinate. Since

$$
\begin{aligned}
-\ln u(t, \mathbf{x}) & =-\ln \left(\frac{e^{\frac{-|\mathbf{x}|^{2}}{4 t}}}{(4 \pi t)^{n / 2}} \int_{\mathbb{R}^{n}} e^{\frac{2 \mathbf{x} \cdot \mathbf{y}-|\mathbf{y}|^{2}}{4 t}} \rho(\mathbf{y}) d \mathbf{y}\right) \\
& =\frac{|\mathbf{x}|^{2}}{4 t}-\ln \left(\int_{\mathbb{R}^{n}} e^{\frac{2 \mathbf{x} \cdot \mathbf{y}-|\mathbf{y}|^{2}}{4 t}} \rho(\mathbf{y}) d \mathbf{y}\right)+\ln (4 \pi t)^{n / 2}
\end{aligned}
$$

then

$$
(-\ln u(t, \mathbf{x}))_{x_{1} x_{1}}=\frac{1}{2 t}-\left(\ln \int_{\mathbb{R}^{n}} e^{\frac{2 \mathbf{x} \cdot \mathbf{y}-|\mathbf{y}|^{2}}{4 t}} \rho(\mathbf{y}) d \mathbf{y}\right)_{x_{1} x_{1}} .
$$


So, the inequality (2.6) is equivalent to prove that

$$
\left(-\ln \int_{\mathbb{R}^{n}} e^{\frac{2 \mathbf{x} \cdot \mathbf{y}-|\mathbf{y}|^{2}}{4 t}} \rho(\mathbf{y}) d \mathbf{y}\right)_{x_{1} x_{1}}=-\frac{\int_{\mathbb{R}^{n}} \frac{y_{1}^{2}}{4 t^{2}} e^{\frac{2 \mathbf{x} \cdot \mathbf{y}-|\mathbf{y}|^{2}}{4 t}} \rho(\mathbf{y}) d \mathbf{y}}{\int_{\mathbb{R}^{n}} e^{\frac{2 \mathbf{x} \cdot \mathbf{y}-|\mathbf{y}|^{2}}{4 t}} \rho(\mathbf{y}) d \mathbf{y}}+\frac{\left(\int_{\mathbb{R}^{n}} \frac{y_{1}}{2 t} e^{\frac{2 \mathbf{x} \cdot \mathbf{y}-|\mathbf{y}|^{2}}{4 t}} \rho(\mathbf{y}) d \mathbf{y}\right)^{2}}{\left(\int_{\mathbb{R}^{n}} e^{\frac{2 \mathbf{x} \cdot \mathbf{y}-|\mathbf{y}|^{2}}{4 t}} \rho(\mathbf{y}) d \mathbf{y}\right)^{2}}<0,
$$

or, equivalently,

$$
-\left(\int_{\mathbb{R}^{n}} \frac{y_{1}^{2}}{4 t^{2}} e^{\frac{2 \mathbf{x} \cdot \mathbf{y}-|\mathbf{y}|^{2}}{4 t}} \rho(\mathbf{y}) d \mathbf{y}\right)\left(\int_{\mathbb{R}^{n}} e^{\frac{2 \mathbf{x} \cdot \mathbf{y}-|\mathbf{y}|^{2}}{4 t}} \rho(\mathbf{y}) d \mathbf{y}\right)+\left(\int_{\mathbb{R}^{n}} \frac{y_{1}}{2 t} e^{\frac{2 \mathbf{x} \cdot \mathbf{y}-|\mathbf{y}|^{2}}{4 t}} \rho(\mathbf{y}) d \mathbf{y}\right)^{2}<0 .
$$

And, for $\mathbf{x}$ fixed, this last inequality is the Cauchy-Schwarz inequality applied to the two functions $g_{1}(\mathbf{y})=1$ and $g_{2}(\mathbf{y})=\frac{y_{1}}{2 t}$ with respect to the auxiliary scalar product

$$
\left\langle g_{1}(\mathbf{y}), g_{2}(\mathbf{y})\right\rangle=\int_{\mathbb{R}^{n}} g_{1}(\mathbf{y}) g_{2}(\mathbf{y}) e^{\frac{2 \mathbf{x} \cdot \mathbf{y}-|\mathbf{y}|^{2}}{4 t}} \rho(\mathbf{y}) d \mathbf{y} .
$$

The strict Cauchy-Schwarz inequality comes from the fact that $\left\langle g_{1}(y)+\lambda g_{2}(y), g_{1}(y)+\lambda g_{2}(y)\right\rangle=$ $\left\langle 1+\lambda \frac{y_{1}}{2 t}, 1+\lambda \frac{y_{1}}{2 t}\right\rangle>0$ for all $\lambda \in \mathbb{R}$. This finishes the proof of $(2.6)$.

To see inequality (2.7), we start with the simplest case when the function $\rho$ is piecewise constant on two half planes. Suppose that the half planes are $x_{1}<0$ and $x_{1}>0$, where the constant values are, respectively, $C^{-}$and $C^{+}\left(C^{-}, C^{+}>0\right)$. The function $u\left(t, x_{1}, x_{2}\right)$ turns out to be independent of $x_{2}$,

$$
u\left(t, x_{1}, x_{2}\right)=\frac{C^{+}+C^{-}}{2}+\frac{C^{+}-C^{-}}{2} \operatorname{erf}\left(\frac{x_{1}}{2 \sqrt{t}}\right),
$$

where $\operatorname{erf}(x)=\frac{2}{\sqrt{\pi}} \int_{0}^{x} e^{-s^{2}} d s$. So, taking $t=1$, in order to obtain inequality (2.7) we only have to care about the $x_{1}$ direction because all derivatives in the $x_{2}$ direction are zero. More exactly, we have to deal with the function of a single variable

$$
h\left(x_{1}\right)=-\ln \left(\frac{C^{+}+C^{-}}{2}+\frac{C^{+}-C^{-}}{2} \operatorname{erf}\left(\frac{x_{1}}{2}\right)\right) .
$$

Since we already know inequality (2.6), we have that $h^{\prime \prime}\left(x_{1}\right)<1 / 2$ for all $x_{1} \in \mathbb{R}$. Then, to conclude that $h^{\prime \prime}\left(x_{1}\right) \leq \beta$ for some $\beta<1 / 2$ it is enough to observe that from the properties of the erf function it follows that $\lim _{x_{1} \rightarrow \pm \infty} h^{\prime \prime}\left(x_{1}\right)=0$.

Now we proceed with the general case of a positive piecewise constant on sectors function $\rho$. From (2.6), with $t=1$, we deduce that at every point the Hessian matrix of $-\ln u\left(1, x_{1}, x_{2}\right)$, as a quadratic form, is strictly less than the quadratic form given by the matrix $\frac{1}{2} I$. So, like in the case studied before, we have to prove strict inequality only near infinity.

For this goal, first at all, let us cover the plane with a finite number of small sectors of the form $\Sigma\left(\alpha_{i}\right)=\left\{\alpha_{i}-\varepsilon \leq \arg \left(x_{1}, x_{2}\right) \leq \alpha_{i}+\varepsilon\right\}$. For each of these sectors, we consider also a second sector $\Sigma^{\prime}\left(\alpha_{i}\right)=\left\{\alpha_{i}-\varepsilon^{\prime} \leq \arg \left(x_{1}, x_{2}\right) \leq \alpha_{i}+\varepsilon^{\prime}\right\}$. The numbers $\varepsilon^{\prime}>\varepsilon>0$ are chosen in such a way that $\sin \varepsilon^{\prime}=2 \sin \varepsilon$, and with the property that in the set $\Sigma^{\prime}\left(\alpha_{i}\right)$ there is at most one discontinuity line of $\rho$.

Now, for each point $\mathbf{x}_{0}$ with $R=\left\|\mathbf{x}_{0}\right\|>0$ and $\arg \left(\mathbf{x}_{0}\right)=\alpha_{i}$, let us consider the discs $B$ and $B^{\prime}$ centered at $\mathbf{x}_{0}$ and with radii $R \sin \varepsilon$ and $R \sin \varepsilon^{\prime}$, respectively. Observe that $B \subset \Sigma\left(\alpha_{i}\right)$ and that $B^{\prime} \subset \Sigma^{\prime}\left(\alpha_{i}\right)$.

With all these conditions, the initial function $\rho$ coincides in $B^{\prime}$ with an auxiliary function $\widetilde{\rho}$ that is either constant or piecewise constant on two half planes. Then, if $u_{\tilde{\rho}}\left(1, x_{1}, x_{2}\right)$ is the solution 
of the heat equation with initial condition $\widetilde{\rho}$, we have already proven that the inequality we are looking for holds for the Hessian matrix of $-\ln \left(u_{\tilde{\rho}}\right)$.

Finally, the following lemma tells us that since $\rho$ and $\widetilde{\rho}$ coincide in $B^{\prime}$, the $\mathcal{C}^{2}(B)$ norms of $u_{\rho}$ and $u_{\widetilde{\rho}}$ are as close as we like if $R$ is sufficiently large. This implies that the inequality is also true for the Hessian matrix of $-\ln u$ on the sectors $\Sigma^{\prime}\left(\alpha_{i}\right)$, at least sufficiently far from the origin, and since there are only finitely many of these sectors, this finishes the proof of the Theorem 3.

Lemma 1. Let $\rho$ and $\widetilde{\rho}$ be two functions with $\|\rho\|_{L^{\infty}\left(\mathbb{R}^{2}\right)},\|\widetilde{\rho}\|_{L^{\infty}\left(\mathbb{R}^{2}\right)} \leq M$ and suppose that $\rho(\mathbf{x})=$ $\widetilde{\rho}(\mathbf{x})$ for all $\mathbf{x}$ in a closed ball of radius $R>0$ centered at $\mathbf{x}_{0}, B_{R}\left(\mathbf{x}_{0}\right)$. Let $u_{\rho}(t, \mathbf{x})$ and $u_{\tilde{\rho}}(t, \mathbf{x})$ be the solutions of the linear heat equation in all the plane with initial condition $\rho$ and $\widetilde{\rho}$ respectively. Then, there exists a constant $K$, depending only on $M$, such that for $t=1$,

$$
\left\|u_{\rho}(1, \cdot)-u_{\widetilde{\rho}}(1, \cdot)\right\|_{\mathcal{C}^{2}\left(B_{R / 2}\left(\mathbf{x}_{0}\right)\right)} \leq K e^{-R^{2} / 64}
$$

Proof: There is no loss of generality in supposing that $\mathbf{x}_{0}=0$ and $\widetilde{\rho} \equiv 0$. It is a simple calculation to see that

$$
\frac{\partial^{2} u_{\rho}\left(1, x_{1}, x_{2}\right)}{\partial x_{1}^{2}}=\frac{1}{4 \pi} \int_{\mathbb{R}^{2} \backslash B_{R}(0)}\left(\frac{\left(x_{1}-y_{1}\right)^{2}}{4}-\frac{1}{2}\right) e^{-\frac{|\mathbf{x}-\mathbf{y}|^{2}}{4}} \rho(\mathbf{y}) d \mathbf{y} .
$$

Then, if $\mathbf{x} \in B_{R / 2}(0)$ and $\mathbf{y} \in \mathbb{R}^{2} \backslash B_{R}(0)$,

$$
\left|\left(\frac{\left(x_{1}-y_{1}\right)^{2}}{4}-\frac{1}{2}\right) e^{-\frac{1 \mathbf{x}-\left.\mathbf{y}\right|^{2}}{4}} \rho(\mathbf{y})\right| \leq M\left(\frac{|\mathbf{x}-\mathbf{y}|^{2}}{4}+\frac{1}{2}\right) e^{-\frac{|\mathbf{x}-\mathbf{y}|^{2}}{16}} e^{\frac{-R^{2}}{64}} e^{-\frac{3|\mathbf{x}-\mathbf{y}|^{2}}{16}} .
$$

and, since $\max _{z>0}\left\{(z+1 / 2) e^{-z / 4}\right\}=4 e^{-7 / 8}$ and $\int_{\mathbb{R}^{2}} e^{-\frac{3|\mathbf{x}-\mathbf{y}|^{2}}{16}} d \mathbf{y}=16 \pi / 3$, it follows that

$$
\frac{\partial^{2} u_{\rho}\left(1, x_{1}, x_{2}\right)}{\partial x_{1}^{2}} \leq K^{\prime} e^{-R^{2} / 64}
$$

for some constant $K^{\prime}$ that depends only on $M$. The estimates for the other second derivatives, for the first derivatives and for the function $u_{\rho}\left(1, x_{1}, x_{2}\right)$ itself are similar.

Remark 2. In order to show that the inequality (2.7) with $\beta<1 / 2$ is sharp, we present here four plots. They correspond to the second derivative of the function $h\left(x_{1}\right)$ defined in (4.8) for $C^{-}=C-1, C^{+}=C+1$ and four different values of $C$. These plots show also that this is really a one-sided inequality, in the sense that $\left|h^{\prime \prime}\left(x_{1}\right)\right|<1 / 2$ cannot be expected to hold in general. 


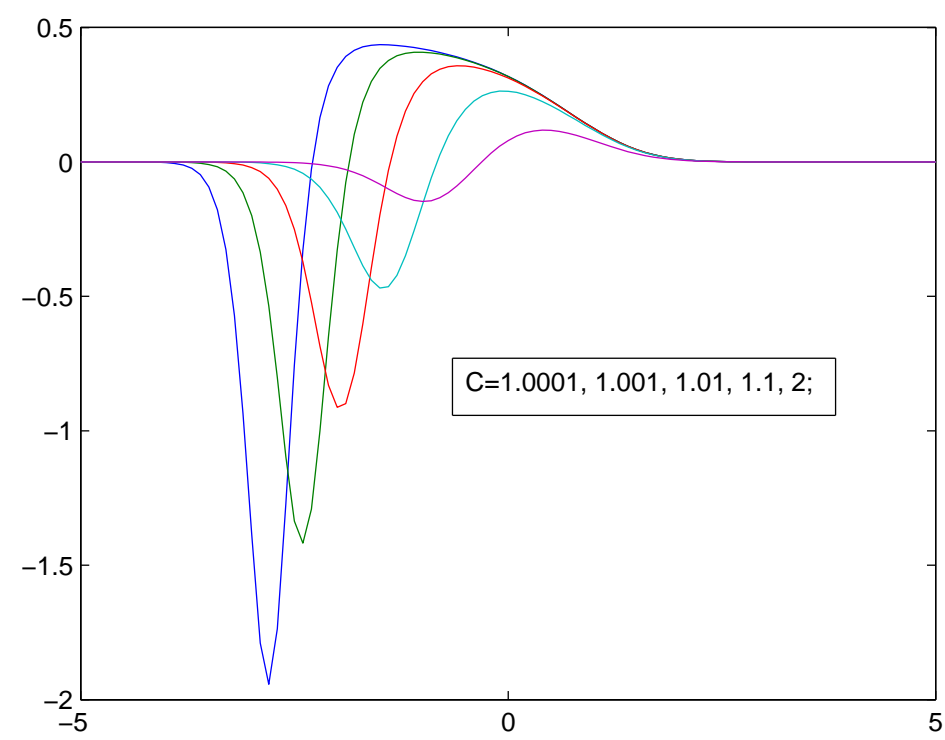

\section{Proof of Theorem 1}

It is known that the solution $u(t, \mathbf{x})$ of the linear heat equation with initial condition $\rho \in L^{\infty}\left(\mathbb{R}^{2}\right)$ and $\inf _{\mathbb{R}^{n}} \rho>0$ satisfies the inequality

$$
|\nabla(\ln u(t, \mathbf{x}))| \leq \frac{K}{\sqrt{t}}
$$

More exactly, if $\rho$ is a positive piecewise constant on sectors function in the plane, it follows that the constant $K$ is

$$
K=\frac{\max \left(C_{1}, C_{2}, \cdots, C_{k}\right)}{2 \pi \min \left(C_{1}, C_{2}, \cdots, C_{k}\right)} .
$$

Then, this upper bound (5.1) proves the validity of the first bound (2.3) of Theorem 2 with $\alpha=1 / 2$.

On the other hand, if $\rho$ is a positive piecewise constant on sectors function in the plane, it is easy to check that if $u(t, \mathbf{x})$ is the solution of the linear heat equation with initial data $\rho$ then, for all $\lambda \in \mathbb{R}, u\left(\lambda^{2} t, \lambda \mathbf{x}\right)$ is also a solution of the same initial value problem. In particular, taking $\lambda=1 / \sqrt{t}$, then $u(1, \mathbf{x} / \sqrt{t})$ is also a solution and, therefore, the initial value problem (2.1) can be written as

$$
\left\{\begin{aligned}
\mathbf{x}^{\prime}(t) & =\frac{-1}{\sqrt{t}} \nabla \tilde{u}\left(\frac{\mathbf{x}(t)}{\sqrt{t}}\right), \quad t>0, \\
\lim _{t \rightarrow 0^{+}} \mathbf{x}(t) & =\mathbf{x}_{0},
\end{aligned}\right.
$$

where $\tilde{u}(\mathbf{x})=\ln u(1, \mathbf{x})$. Then, the second bound of Theorem 2 and, therefore, the proof of Theorem 1 , are obtained from the estimate (2.7) of Theorem 3.

\section{REFERENCES}

[1] Agarwal, R. P., Lakshmikantham, V. (1993). Uniqueness and nonuniqueness criteria for ordinary differential equations, World Scientific. 
[2] Avinyó, A., Solà-Morales, J., València, M. (1999). Transformations with a given Jacobian. In Montenegro R. et al. Eds., Actas XVI CEDYA, Congreso de Ecuaciones Diferenciales y Aplicaciones, VI CMA, Congreso de Matemática Aplicada. Servicio de Publicaciones y Producción Documental de la Universidad de Las Palmas de Gran Canaria. Las Palmas de Gran Canaria (Spain). 481-487

[3] Avinyó, A., Solà-Morales, J., València, M. (2003) On maps with given Jacobians involving the heat equation. Z. Angew. Math. Phys. 54 (2003), no. 6, 919-936.

[4] Avinyó, A., Solà-Morales, J., València, M. On the diffusion algorithm for density-equalizing maps with piecewise constant initial data. Submitted to Mathematical Methods to Applied Sciences

[5] Belonosov V. S. (1979). Estimates of solutions of parabolic systems in weighted Hölder classes and some of their applications. Mat. Sb., 110. 163-188. English Trans: Math. USSR Sb. 38, (1979), 151-173.

[6] Carrillo J. A., Lisini. S. (2010). On the asymptotic behavior of the gradient flow of a polyconvex functional. In Holden, H., Karslen, K. Eds., Contemporary Mathematics series AMS. 526, 37-51

[7] Dacorogna B. (1998). Direct Methods in the Calculus of Variations, Springer-Verlag.

[8] Dorling D. , Newman M. E. J., Barford A., (2008). The Atlas of the Real World. Mapping the way we live, Thames \& Hudssos.

[9] Gastner, M..; Newman, M. E. J. (2004). Diffusion-based method for producing density-equalizing maps. Proc. Natl. Acad. Sci. USA 101 , no. 20, 7499-7504.

[10] Hartman, P., (1982), Ordinary Differential Equations, Birkhäuser.

[11] Henry, D., (1981). Geometric theory of semilinear parabolic equations, Lecture Notes in Math., 840, SpringerVerlag.

[12] Mora X., (1983). Semilinear parabolic problems define semiflows on $C^{k}$ spaces, Trans. Amer. Math. Soc. 278, $21-55$.

[13] Lekien, F., Leonard, N. E., (2009), Non-uniform coverage and cartograms. SIAM. J. Control Optim. 49, Issue $1,351-372$.

[14] Nagumo, N., (1926). Eine hinreichende Bendingung für die Unität der Lösung von Differentialgleichungen erster Ordnung Japan J. Math. 3, 107-112.

[15] Russo G. , (1990). Deterministic diffusion of particles. Comm. Pure Appl. Math., XLIII, 697-733. 